\title{
Coronas cementadas comparado con coronas atornilladas para pacientes con restauraciones fijas implantosoportadas.
}

\section{Cement-retained versus screw-retained crowns in patients with implant rehabilitation.}

Alexandra Bayer ${ }^{1}$, Javiera Martínez ${ }^{1}$, Javiera Valenzuela ${ }^{1}$, Sebastián Prado ${ }^{1 *}$

\begin{abstract}
RESUMEN
Introducción: Los implantes dentales se han transformado en una opción de tratamiento de suma relevancia para pacientes parcial o totalmente desdentados. EI éxito del tratamiento puede verse afectado por la elección del tipo de retención de estos (cementada o atornillada). A pesar que ambas presentan ventajas, aún no existe consenso sobre el mejor tipo de retención para restauraciones fijas implantosoportadas. Métodos: Realizamos una búsqueda en Epistemonikos, la mayor base de datos de revisiones sistemáticas en salud, la cual es mantenida mediante el cribado de múltiples fuentes de información, incluyendo MEDLINE, EMBASE, Cochrane, entre otras. Extrajimos los datos desde las revisiones identificadas, analizamos los datos de los estudios primarios, realizamos un metanálisis y preparamos una tabla de resumen de los resultados utilizando el método GRADE. Resultados y conclusiones: Identificamos 14 revisiones sistemáticas que en conjunto incluyeron 43 estudios primarios, de los cuales cinco corresponden a ensayos aleatorizados. De estos, solamente dos ensayos responden a la pregunta de interés de manera directa. Concluimos que las coronas atornilladas podrían aumentar levemente el riesgo de pérdida de implante a largo plazo, podrían resultar en nula o poca diferencia en el riesgo de pérdida de implante a mediano plazo, reabsorción ósea y periimplantitis, pero la certeza de evidencia ha sido evaluada como baja. Por otro lado, no es posible establecer con claridad si las coronas cementadas disminuyen el riesgo de complicaciones estéticas y protésicas, ya que la certeza de la evidencia existente ha sido evaluada como muy baja.
\end{abstract}

\section{PALABRAS CLAVE}

Implante dental; Corona atornillada; Corona cementada; Epistemonikos; GRADE.

\begin{abstract}
Introduction: Dental implants have become a highly relevant treatment option for partially or totally edentulous patients. Implant retention systems (cemented or screwed) can influence the treatment success. Although both have advantages, there is still no consensus on the best type of retention for implant-supported fixed restorations. Methods: We searched in Epistemonikos, the largest database of systematic reviews in health, which is maintained by screening multiple information sources, including MEDLINE, EMBASE, Cochrane, among others. We extracted data from the systematic reviews, reanalyzed data of primary studies, conducted a metaanalysis and generated a summary of findings table using the GRADE approach. Results and conclusions: We identified 14 systematic reviews including 43 primary studies overall, of which five were randomized trials. Of these, only two trials answer the question of interest. We concluded that screw-retained crowns may increase longterm implant loss, may make little or no difference in the risk of medium-term implant loss, bone resorption, and peri-implantitis, but the certainty of the evidence has been assessed as low. On the other hand, it is not possible to clearly establish whether cemented crowns reduce the risk of cosmetic and prosthetic complications, since the certainty of the evidence has been assessed as very low.
\end{abstract}

\section{KEY WORDS}

Dental implant; Screw-retained; Cement-retained; Epistemonikos; GRADE.

Int. J. Inter. Dent Vol. 14(1); 83-88, 2021. 


\section{PROBLEMA}

En la actualidad, los implantes dentales se han transformado en una opción de tratamiento de suma relevancia para pacientes desdentados parciales o totales, ayudando en la mejora de su calidad de vida. No obstante, este procedimiento puede presentar diversas complicaciones que pueden verse afectadas por decisiones como el tipo de retención de la restauración del implante. Este puede ser cementado o atornillado ${ }^{[1]}$.

La elección entre prótesis de implantes atornilladas y cementadas sigue siendo un tema discutido entre los odontólogos. Ambos tipos de retención tienen ventajas y limitaciones en cuanto a la facilidad de fabricación, estética, pérdida de retención, recuperabilidad, efecto sobre el tejido periimplantario, fractura de porcelana y rendimiento clínico. Se ha reportado que las coronas cementadas presentan mejores resultados estéticos y menores complicaciones técnicas, sin embargo, estudios han descrito mayores complicaciones biológicas en éstas ${ }^{[2]}$. Por otro lado, estudios han descrito una mejor capacidad de recuperación y un mejor control de la salud de los tejidos blandos para coronas atornilladas ${ }^{[2]}$, pero una mayor tasa de problemas técnicos ${ }^{[3]}$.

Debido a que aún existe incertidumbre sobre el mejor tipo de retención para restauraciones fijas implantosoportadas, este resumen busca comparar el comportamiento clínico de ambos sistemas de retención.

\section{MÉTODOS}

Realizamos una búsqueda en Epistemonikos, la mayor base de datos de revisiones sistemáticas en salud, la cual es mantenida mediante búsquedas en múltiples fuentes de información, incluyendo MEDLINE, EMBASE, Cochrane, entre otras. Extrajimos los datos desde las revisiones identificadas y analizamos los datos de los estudios primarios. Con esta información, generamos un resumen estructurado denominado FRISBEE (Friendly Summaries of Body of Evidence using Epistemonikos), siguiendo un formato preestablecido, que incluye mensajes clave, un resumen del conjunto de evidencia (presentado como matriz de evidencia en Epistemonikos), metanálisis del total de los estudios cuando sea posible, una tabla de resumen de resultados con el método GRADE y una sección de otras consideraciones para la toma de decisión.

\section{Mensajes clave}

- Las coronas atornilladas podrían aumentar levemente la pérdida de implante a largo plazo (certeza de evidencia baja)

- Las coronas cementadas podrían resultar en nula o poca diferencia en el riesgo de pérdida de implante a mediano plazo, reabsorción ósea y periimplantitis (certeza de evidencia baja).

- No es posible establecer con claridad si las coronas cementadas disminuyen el riesgo de complicaciones estéticas y protésicas, debido a que la certeza de la evidencia existente ha sido evaluada como muy baja.
Acerca del conjunto de evidencia para esta pregunta

Encontramos 14 revisiones sistemáticas $^{[1-14]}$ que incluyeron cinco
estudios primarios reportados en 46
referencias $^{[15-60]}$, de los cuales, cinco
son ensayos aleatorizados reportados
en seis referencias

Cuál es la evidencia

De estos, solamente dos ensayos,

Véase matriz de evidencia en Epistemonikos más abajo. reportados en tres referencias ${ }^{[15,20,40]}$, responden a la pregunta de interés. El resto de los ensayos fueron excluidos ya que comparaban otros tratamientos relacionados a los implantes dentales.

Esta tabla y el resumen en general se basan en estos últimos, dado que los estudios observacionales no aumentaban la certeza de la evidencia existente, ni entregaban información adicional relevante.

La edad de los participantes varió entre 27 a 77 años entre los ensayos $^{[15,20]}$.

Uno de los ensayos incluyó pacientes que requerían implantes con coronas unitarias en la zona de caninos,

Qué tipo de pacientes incluyeron los estudios* premolares y molares ${ }^{[15,40]}$. El otro ensayo incluyó pacientes que requerían rehabilitación con implantes de arco completo ${ }^{[20]}$

No se reportó información sobre la presencia de enfermedades sistémicas y/o hábito tabáquico en los participantes.

Todos los ensayos compararon el uso de coronas metal-cerámicas cementadas contra coronas atornilladas para rehabilitación sobre implantes dentales.

Qué tipo de intervenciones incluyeron los estudios*

Ambos ensayos utilizaron para las coronas cementadas cemento temporal sin eugenol, a base de óxido de zinc (Temp Bond NE) $)^{[15,20,40]}$.

El sistema de los implantes variaron entre los ensayos incluidos. Uno utilizó un sistema hexagonal externo ${ }^{[15,}$ ${ }^{40]}$, mientras que el otro un sistema hexagonal interno ${ }^{[20]}$.

Los ensayos reportaron múltiples desenlaces, los cuales fueron agrupados por las revisiones sistemáticas de la siguiente manera:

- Pérdida de implante a largo plazo

- Pérdida de implante a mediano

Qué tipo de desenlaces plazo midieron

- Complicaciones protésicas

- Complicaciones estéticas

- Periimplantitis

- Reabsorción ósea

El seguimiento de los ensayos varió entre cuatro y diez años ${ }^{[15,20,40]}$.

* La información sobre los estudios primarios es extraída desde las revisiones sistemáticas identificadas, no directamente desde los estudios, a menos que se especifique lo contrario. 


\section{RESUMEN DE LOS RESULTADOS}

La información sobre los efectos del uso de coronas cementadas comparado con coronas atornilladas en pacientes con rehabilitación sobre implantes está basada en dos ensayos aleatorizados que incluyeron 328 implantes dentales (46 pacientes) $)^{[15,20,40]}$.

Dos ensayos midieron el desenlace pérdida de implante a largo plazo (304 implantes $)^{[15,20,40]}$. Dos ensayos midieron el desenlace complicaciones protésicas (58 implantes) ${ }^{[15,20,40]}$. Dos ensayos midieron el desenlace reabsorción ósea (304 implantes) ${ }^{[15,20,40]}$. Ninguna revisión permitió la extracción de datos del desenlace pérdida de implante a mediano plazo, complicaciones estéticas y periimplantitis, por lo que la información de dicho desenlace se presenta como síntesis narrativa.

El resumen de los resultados es el siguiente:

- Las coronas atornilladas comparadas con las coronas cementadas podrían aumentar levemente la pérdida de implante a largo plazo (certeza de la evidencia baja).

- Las coronas cementadas comparadas con las coronas atornilladas podría resultar en poca o nula diferencia en la pérdida de implante a mediano plazo (certeza de la evidencia baja).

- Las coronas cementadas comparadas con las coronas atornilladas podría resultar en poca o nula diferencia en la reabsorción ósea (certeza de la evidencia baja).

- No es posible establecer con claridad si las coronas cementadas comparadas con las coronas atornilladas disminuye el riesgo de complicaciones protésicas, debido a que la certeza de la evidencia existente ha sido evaluada como muy baja.

- No es posible establecer con claridad si las coronas cementadas disminuyen el riesgo de complicaciones estéticas, debido a que la certeza de la evidencia existente ha sido evaluada como muy baja.

- Las coronas cementadas comparadas con las coronas atornilladas podría resultar en poca o nula diferencia en el riesgo de periimplantitis (certeza de la evidencia baja).

\section{Coronas cementadas comparado con restauraciones fijas implantosoportadas \\ coronas atornilladas en pacientes con}

Pacientes

Intervención Coronas cementadas

Comparación Coronas atornilladas

\begin{tabular}{|c|c|c|c|c|}
\hline \multirow[t]{3}{*}{ Desenlaces } & \multicolumn{2}{|c|}{ Efecto absoluto* } & \multirow{3}{*}{$\begin{array}{l}\text { Efecto } \\
\text { relativo } \\
\text { (IC 95\%) }\end{array}$} & \multirow{3}{*}{$\begin{array}{l}\text { Certeza de } \\
\text { la evidencia } \\
\text { (GRADE) }\end{array}$} \\
\hline & $\begin{array}{c}\text { CON } \\
\text { Coronas atornilladas }\end{array}$ & $\begin{array}{l}\text { CON } \\
\text { Coronas cementadas }\end{array}$ & & \\
\hline & \multicolumn{2}{|c|}{ Diferencia: implantes por 1000} & & \\
\hline \multirow{2}{*}{$\begin{array}{c}\text { Pérdida de } \\
\text { implante a largo } \\
\text { plazo } \\
\text { (8 a } 10 \text { años) }\end{array}$} & 20 por 1000 & 10 por 1000 & \multirow{2}{*}{$\begin{array}{l}\text { RR } 0,49 \\
(0,07 \mathrm{a} \\
3,67)\end{array}$} & \multirow{2}{*}{$\underset{\text { Baja }}{\oplus \bigcirc^{1,2}}$} \\
\hline & \multicolumn{2}{|c|}{$\begin{array}{l}\text { Diferencia: } 10 \text { menos } \\
\text { (Margen de error: de } 18 \text { menos a } 53 \text { más) }\end{array}$} & & \\
\hline $\begin{array}{l}\text { Pérdida de } \\
\text { implante a } \\
\text { mediano plazo } \\
(<5 \text { años })\end{array}$ & \multicolumn{2}{|c|}{$\begin{array}{l}\text { Un ensayo [40] (24 implantes) reportó que no } \\
\text { hubo eventos de fracaso de implante durante el } \\
\text { periodo de seguimiento. }\end{array}$} & -- & $\underset{\mathrm{Baja}}{\oplus \oplus \bigcirc^{1,2}}$ \\
\hline \multirow[t]{2}{*}{$\begin{array}{l}\text { Reabsorción } \\
\text { ósea** }\end{array}$} & $0,48 \mathrm{~mm}$ & $0,38 \mathrm{~mm}$ & \multirow[t]{2}{*}{--} & \multirow{2}{*}{$\underset{\text { Baja }}{\oplus \bigcirc^{1,3}}$} \\
\hline & \multicolumn{2}{|c|}{$\begin{array}{l}\text { DM: } 0,10 \text { milímetros menos } \\
\text { (Margen de error: } 0,24 \text { menos a } 0,05 \text { más) }\end{array}$} & & \\
\hline \multirow[t]{2}{*}{$\begin{array}{l}\text { Complicaciones } \\
\text { protésicas*** }\end{array}$} & 138 por 1000 & 207 por 1000 & \multirow{2}{*}{$\begin{array}{l}\text { RR } 1,50 \\
(0,51 \text { to } \\
4,38)\end{array}$} & \multirow{2}{*}{$\begin{array}{l}\oplus \bigcirc \bigcirc \bigcirc^{1,2} \\
\text { Muy baja }\end{array}$} \\
\hline & \multicolumn{2}{|c|}{$\begin{array}{l}\text { Diferencia: } 69 \text { más } \\
\text { (Margen de error: de } 68 \text { menos a } 466 \text { más) }\end{array}$} & & \\
\hline
\end{tabular}




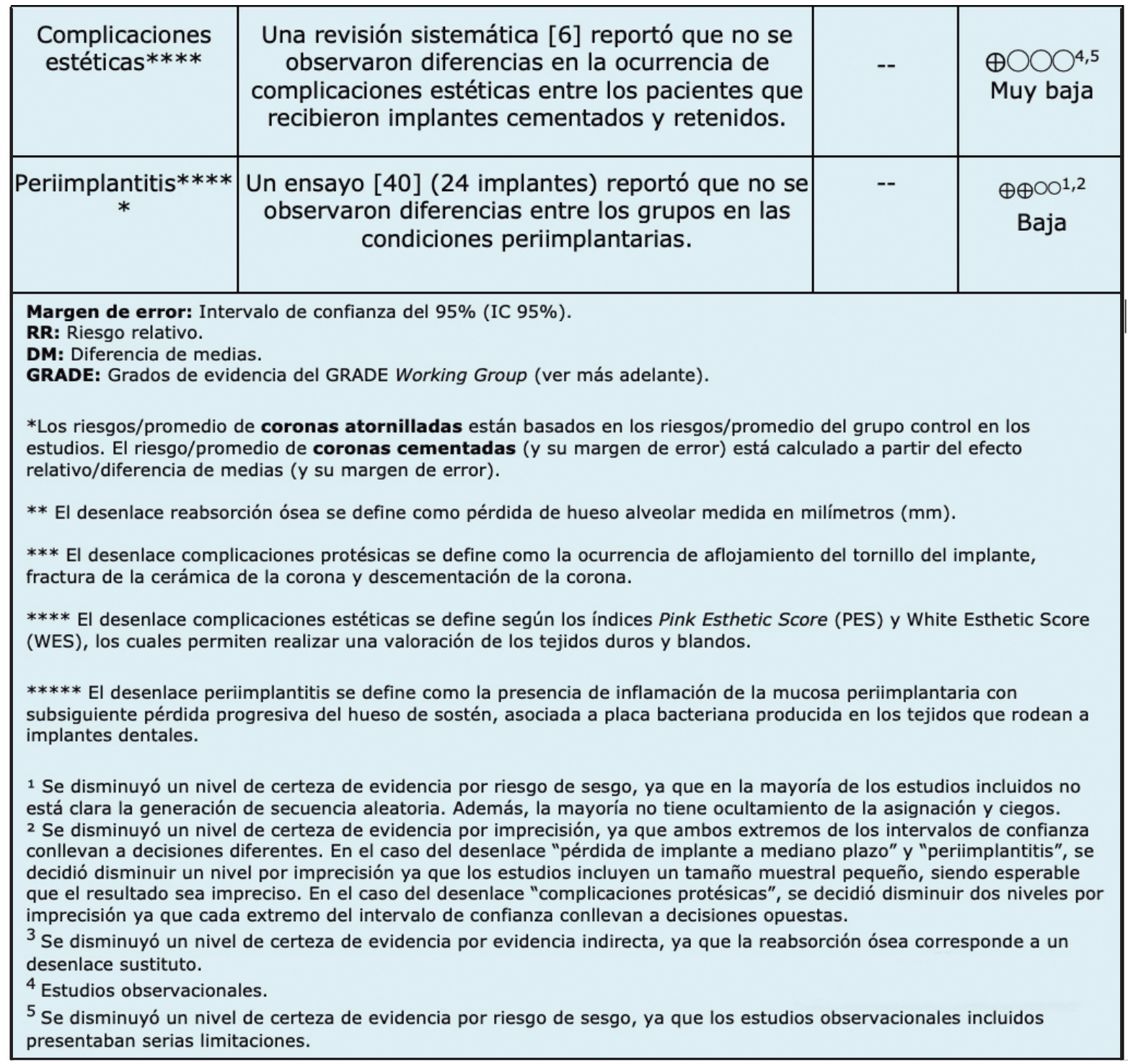

Siga el enlace para acceder a la versión interactiva de esta tabla (Interactive Summary of Findings - iSoF)

\begin{tabular}{l}
\hline Acerca de la certeza de la evidencia (GRADE)* \\
A $\oplus \bigoplus \bigoplus$ \\
que el efecto sea sustancialmente distinto' es baja. \\
$\bigoplus \bigoplus \bigoplus \bigcirc$ \\
Moderada: La investigación entrega una buena indicación del efecto probable. La probabilidad de \\
que el efecto sea sustancialmente distintoł es moderada. \\
$\bigoplus \bigoplus \bigcirc \bigcirc$ \\
Baja: La investigación entrega alguna indicación del efecto probable. Sin embargo, la probabilidad \\
de que el efecto sea sustancialmente distinto† es alta. \\
$\bigoplus \bigcirc \bigcirc \bigcirc$ \\
Muy baja: La investigación no entrega una estimación confiable del efecto probable. La \\
probabilidad de que el efecto sea sustancialmente distinto† es muy alta. \\
*Esto es también denominado 'calidad de la evidencia' o 'confianza en los estimadores del efecto'. \\
tSustancialmente distinto = una diferencia suficientemente grande como para afectar la decisión \\
\hline
\end{tabular}




\section{OTRAS CONSIDERACIONES PARA LA TOMA DE DECISIÓN}

\section{A quién se aplica y a quién no se aplica esta evidencia}

- Los resultados de este resumen son aplicables a pacientes adultos ASA I o II con rehabilitación sobre implantes.

- Esta evidencia no aplicaría para pacientes fumadores, con enfermedad periodontal, consumidores de bifosfonatos 0 con enfermedades sistémicas no controladas.

\section{Sobre los desenlaces incluidos en este resumen}

- Los desenlaces seleccionados son considerados críticos para la toma de decisión de acuerdo a la opinión de los autores de este resumen, coincidiendo en general con los evaluados por las revisiones sistemáticas identificadas.

- El desenlace reabsorción ósea fue incluido en la tabla de resumen de resultados debido a que se trata de un desenlace relevante para los expertos clínicos, aún cuando se trata de un desenlace sustituto de periimplantitis.

\section{Balance daño/beneficio y certeza de la evidencia}

- La evidencia muestra un posible beneficio con respecto a la pérdida de implante a largo plazo de las coronas cementadas en comparación a las coronas atornilladas. Sin embargo, la certeza ha sido evaluada como baja.

- Por otro lado, existe incertidumbre sobre el efecto del uso de coronas cementadas en relación al riesgo de desarrollar complicaciones protésicas y estéticas, ya que la evidencia ha sido evaluada como muy baja.

- De acuerdo a lo anterior, no es posible hacer un adecuado balance entre daños y beneficios entre los distintos tipos de retención debido a la incertidumbre existente asociadas a las limitaciones de la evidencia existente.

\section{Consideraciones de recursos}

- La confección de restauraciones cementadas en comparación a las atornilladas tienen costos similares, sin embargo, la confección de restauraciones cementadas es más fácil debido a las técnicas de laboratorio utilizadas y no requieren de componentes adicionales necesarios para su fabricación que aumenten el costo de éstas como tornillos de fijación que si son utilizados en la fabricación de coronas atornilladas.

- Por otro lado, las coronas atornilladas permiten una reparación más sencilla en el caso de fallas en el implante, pudiendo ser esto una ventaja costo- beneficio a largo plazo para las coronas atornilladas

\section{Qué piensan los pacientes y sus tratantes}

- Entre los profesionales existe discrepancia en cuanto a las recomendaciones sobre el uso de coronas cementadas y atornilladas. Por lo general, suele ser una decisión clínica basada en la condición oral del paciente y las preferencias del tratante, debido a la incertidumbre en relación a la evidencia existente.

- Los pacientes por lo general no muestran preferencias por un tipo específico de retención.

\section{Diferencias entre este resumen y otras fuentes}

- En cuanto a los resultados, las conclusiones de este resumen concuerdan con tres revisiones sistemáticas identificadas ${ }^{[3,11,12]}$, las cuales consideran que las coronas cementadas disminuyen la pérdida de implante a largo plazo.

- Las conclusiones de este resumen no concuerdan con cuatro RS identificadas ${ }^{[2,3,7,11]}$, las cuales consideran que las coronas cementadas disminuyen las complicaciones protésicas.

- Las conclusiones de este resumen no concuerdan con seis RS identificadas ${ }^{[1-4,11,13]}$, las cuales consideran que las coronas cementadas aumentan la reabsorción ósea.

- Sin embargo, ninguna de las revisiones sistemáticas realizó una evaluación formal de la certeza de la evidencia en relación a cada uno de estos desenlaces.

\section{¿Puede que cambie esta información en el futuro?}

- La probabilidad de que una futura evidencia cambie las conclusiones de este resumen es alta, dada la incertidumbre de la evidencia existente.

- Luego de realizar una búsqueda en la International Clinical Trials Registry Platform de la Organización Mundial de la Salud y en la base de datos PROSPERO, no se identificaron registros de ensayos clínicos ni revisiones sistemáticas relacionados con la pregunta de investigación.

\section{CÓMO REALIZAMOS ESTE RESUMEN}

Mediante métodos automatizados y colaborativos recopilamos toda la evidencia relevante para la pregunta de interés y la presentamos en una matriz de evidencia.

\section{Siga el enlace para acceder a la versión interactiva:} Coronas cementadas versus coronas atornilladas en pacientes con rehabilitación sobre implantes.

\section{NOTAS}

Si con posterioridad a la publicación de este resumen se publican nuevas revisiones sistemáticas sobre este tema, en la parte superior de la matriz se mostrará un aviso de "nueva evidencia".

Este artículo es parte del proyecto síntesis de evidencia de Epistemonikos. Se elabora con una metodología preestablecida, siguiendo rigurosos estándares metodológicos y proceso de revisión por pares interno. Cada uno de estos artículos corresponde a un resumen, denominado FRISBEE (Friendly Summary of Body of Evidence using Epistemonikos), cuyo principal objetivo es sintetizar el conjunto de evidencia de una pregunta específica, en un formato amigable a los profesionales clínicos. Sus principales recursos se basan en la matriz de evidencia de Epistemonikos y análisis de resultados usando metodología GRADE. Mayores detalles de los métodos para elaborar este FRISBEE están descritos aquí (http://dx.doi.org/10.5867/medwave.2014.06.5997)

La Fundación Epistemonikos es una organización que busca acercar la información a quienes toman decisiones en salud, mediante el uso de tecnologías. Su principal desarrollo es la base de datos Epistemonikos (www.epistemonikos.org).

\section{DECLARACIÓN DE CONFLICTOS DE INTERESES}

Los autores declaran no tener conflictos de intereses con la materia de este artículo.

\section{AGRADECIMIENTOS}

Este resumen de evidencia fue elaborado con el apoyo metodológico del Centro Evidencia UC, Facultad de Medicina, Pontificia Universidad Católica de Chile.

\section{Bibliografía}

1. Al-Fahd AAN, Alsourori A, Al-Qutabi AY, Farouk M, Abbas N. Impact of screw retained versus cement retained implant-supported prosthesis on peri-implantitis: $A$ systematic review and meta-analysis. Int Dent Med J Adv Res. 2015;1(1):1-6.

2. Ragauskaitè A, Žekonis G, Žilinskas J, Gleiznys A, Ivanauskienè E, Gleiznys D. The comparison of cement- and screw-retained crowns from technical and biological points of view. Stomatologija. 2017;19(2):44-50.

3. Sailer I, Mühlemann S, Zwahlen M, Hämmerle CHF, Schneider D. Cemented and screw-retained implant reconstructions: A systematic review of the survival and complication rates. Clin Oral Implants Res. 2012;23(SUPPL.6):163-201.

4. Staubli N, Walter C, Schmidt JC, Weiger R, Zitzmann NU. Excess cement and the risk of peri-implant disease - a systematic review. Clin Oral Implants Res. 2017;28(10):1278-90.

5. De Brandão ML, Vettore M V, Vidigal Júnior GM. Peri-implant bone loss in cement- and screw-retained prostheses: Systematic review and meta-analysis. J
Clin Periodontol. 2013;40(3):287-95.

6. Martin W, Pollini A, Morton D. The influence of restorative procedures on esthetic outcomes in implant dentistry: a systematic review. Int J Oral Maxillofac Implants. 2014;29:142-54

7. Jain JK, Sethuraman R, Chauhan S, Javiya P, Srivastava S, Patel R, et al. Retention failures in cement- and screw-retained fixed restorations on dental implants in partially edentulous arches: A systematic review with meta-analysis Jatin. J Indian Prosthodont Soc. 2018;18(3):201-11.

8. Salvi GE, Brägger U. Mechanical and technical risks in implant therapy. Int J Oral Maxillofac Implants. 2009;24:69-85.

9. Sherif S, Susarla HK, Kapos T, Munoz D, Chang BM, Wright RF. A systematic review of screw- versus cement-retained implant-supported fixed restorations. J Prosthodont. 2014;23(1):1-9.

10. Ma S, Fenton A. Screw- versus cement-retained implant prostheses: a systematic 


\section{Bibliografía}

review of prosthodontic maintenance and complications. Int $\mathrm{J}$ Prosthodont. 2015;28(2):127-45

11. Lemos CAA, De Souza Batista VE, Almeida DADF, Santiago Júnior JF, Verr FR, Pellizzer EP. Evaluation of cement-retained versus screw-retained implantsupported restorations for marginal bone loss A systematic review and metaanalysis. J Prosthet Dent. 2016;115(4):419-27.

12. Weber H-P, Sukotjo C. Does the type of implant prosthesis affect outcomes in the partially edentulous patient? Int J Oral Maxillofac Implants. 2007;22:140-72. 13. Serino G, Hultin K. Periimplant disease and prosthetic risk indicators: a literature review. Implant Dent. 2019;28(2):125-37.

14. Chrcanovic BR, Albrektsson T, Wennerberg A. Reasons for failures of oral implants. J Oral Rehabil. 2014;41(6):443-76.

15. Vigolo P, Mutinelli S, Givani A, Stellini E. Cemented versus screw-retained implant-supported single-tooth crowns: a 10-year randomised controlled trial. Eur J Oral Implantol. 2012;5(4):355-64

16. De la Rosa M, Rodríguez A, Sierra K, Mendoza G, Chambrone L. Predictors of peri-implant bone loss during long-term maintenance of patients treated with $10-\mathrm{mm}$ implants and single crown restorations. Int J Oral Maxillofac Implants. 2013;28(3):798-802

17. Kotsakis GA, Zhang L, Gaillard P, Raedel M, Walter MH, Konstantinidis IK Investigation of the association between cement retention and prevalent peri-implant diseases: a cross-sectional study. J Periodontol. 2016;87(3):212-20.

18. Brägger U, Aeschlimann S, Bürgin W, Hämmerle CH, Lang NP. Biological and technical complications and failures with fixed partial dentures (FPD) on implants and teeth after four to five years of function. Clin Oral Implants Res. 2001;12(1):2634.

19. Mericske-Stern R, Gütter L, Rösch R, Mericske E. Clinical evaluation and prosthetic complications of single tooth replacements by non-submerged implants. Clin Oral Implants Res. 2001;12(4):309-18.

20. Crespi R, Capparè P, Gastaldi G, Gherlone EF. Immediate occlusal loading of full-arch rehabilitations: screw-retained versus cement-retained prosthesis. An 8-year clinical evaluation. Int J Oral Maxillofac Implants. 2014;29(6):1406-11.

21. Cicciù M, Beretta M, Risitano G, Maiorana C. Cemented-retained vs screwretained implant restorations: an investigation on 1939 dental implants. Minerva Stomatol. 2008;57(4):167-79.

22. Kemppainen P, Eskola S, Ylipaavalniemi P. A comparative prospective clinica study of two single-tooth implants: a preliminary report of 102 implants. J Prosthet Dent. 1997;77(4):382-7.

23. Weber HP, Kim DM, Ng MW, Hwang JW, Fiorellini JP. Peri-implant soft-tissue health surrounding cement- and screw-retained implant restorations: a multi-center, 3-year prospective study. Clin Oral Implants Res. 2006;17(4):375-9

24. McMillan AS, Allen PF, Bin Ismail I. A retrospective multicenter evaluation of single tooth implant experience at three centers in the United Kingdom. J Prosthet Dent. 1998;79(4):410-4.

25. Hof M, Pommer B, GD S, Sütö D, Watzek G, Zechner W. Esthetic evaluation of single-tooth implants in the anterior maxilla following autologous bone augmentation Clin Oral Implants Res. 2013;24 Suppl A:88-93.

26. Brägger U, Karoussis I, Persson R, Pjetursson B, Salvi G, Lang N. Technica and biological complications/failures with single crowns and fixed partial dentures on implants: a 10-year prospective cohort study. Clin Oral Implants Res. 2005;16(3):326-34

27. Nedir R, Bischof M, Szmukler-Moncler S, Belser UC, Samson J. Prosthetic complications with dental implants: from an up-to-8-year experience in private practice. Int J Oral Maxillofac Implants. 2006;21(6):919-28.

28. Nissan J, Narobai D, Gross O, Ghelfan O, Chaushu G. Long-term outcome of cemented versus screw-retained implant-supported partial restorations. Int J Oral Maxillofac Implants. 2011;26(5):1102-7.

29. Ferreiroa A, Peñarrocha-Diago M, Pradíes G, MF S-R, Agustín-Panadero R Cemented and screw-retained implant-supported single-tooth restorations in the molar mandibular region: A retrospective comparison study after an observation period of 1 to 4 years. J Clin Exp Dent. 2015;7(1):e89-94

30. Linkevicius T, Puisys A, Vindasiute E, Linkeviciene L, Apse P. Does residual cement around implant-supported restorations cause peri-implant disease? A retrospective case analysis. Clin Oral Implants Res. 2013;24(11):1179-84.

31. Schropp L, Kostopoulos L, Wenzel A, Isidor F. Clinical and radiographic performance of delayed-immediate single-tooth implant placement associated with peri-implant bone defects. A 2-year prospective, controlled, randomized follow-up report. J Clin Periodontol. 2005;32(5):480-7.

32. Wannfors K, Smedberg JI. A prospective clinical evaluation of different singletooth restoration designs on osseointegrated implants. A 3-year follow-up of Brånemark implants. Clin Oral Implants Res. 1999;10(6):453-8.

33. Woelber JP, Ratka-Krueger P, Vach K, Frisch E. Decementation rates and the peri-implant tissue status of implant-supported fixed restorations retained via zinc oxide cement: a retrospective 10-23-year study. Clin Implant Dent Relat Res. 2016;18(5):917-25

34. Sherif S, Susarla SM, Hwang JW, Weber HP, Wright RF. Clinician- and patientreported long-term evaluation of screw- and cement-retained implant restorations: a 5-year prospective study. Clin Oral Investig. 2011;15(6):993-9.

35. Henriksson K, Jemt T. Evaluation of custom-made procera ceramic abutments for single-implant tooth replacement: a prospective 1 -year follow-up study. Int
Prosthodont. 2004;16(6):626-30

36. Krennmair G, Schmidinger S, Waldenberger O. Single-tooth replacement with the Frialit-2 system: a retrospective clinical analysis of 146 implants. Int J Oral Maxillofac Implants. 2002;17(1):78-85

37. Santing HJ, Raghoebar GM, Vissink A, den Hartog L, Meijer HJ. Performance of the Straumann Bone Level Implant system for anterior single-tooth replacements in augmented and nonaugmented sites: a prospective cohort study with 60 consecutive patients. Clin Oral Implants Res. 2013;24(8):941-8.

38. Drago CJ, Lazzara RJ. Immediate occlusal loading of Osseotite implants in mandibular edentulous patients: a prospective observational report with 18-month data. J Prosthodont. 2006;15(3):187-94.

39. Jemt T. Cemented CeraOne and porcelain fused to TiAdapt abutment singleimplant crown restorations: a 10-year comparative follow-up study. Clin Implant Dent Relat Res. 2009;11(4):303-10.

40. Vigolo P, Givani A, Majzoub Z, Cordioli G. Cemented versus screw-retained implant-supported single-tooth crowns: a 4-year prospective clinical study. Int J Oral Maxillofac Implants. 2004;19(2):260-5

41. Duncan JP, Nazarova E, Vogiatzi T, Taylor TD. Prosthodontic complications in a prospective clinical trial of single-stage implants at 36 months. Int J Oral Maxillofac Implants. 2003;18(4):561-5.

42. Levine RA, Clem D, Beagle J, Ganeles J, Johnson P, Solnit G. et al. Multicenter retrospective analysis of the solid-screw ITI implant for posterior single-tooth replacements. Int J Oral Maxillofac Implants. 2002;17(4):550-6.

43. Priest $\mathrm{G}$. Single-tooth implants and their role in preserving remaining teeth: a 10-year survival study. Int J Oral Maxillofac Implants. 1999;14(2):181-8.

44. Zembic A, Bösch A, Jung RE, Hämmerle CH, Sailer I. Five-year results of a randomized controlled clinical trial comparing zirconia and titanium abutments supporting single-implant crowns in canine and posterior regions. Clin Oral Implants Res. 2013;24(4):384-90.

45. Cutrim ES, Peruzzo DC, Benatti B. Evaluation of soft tissues around single tooth implants in the anterior maxilla restored with cemented and screw-retained crowns. J Oral Implantol. 2012;38(6):700-5

46. Linkevicius T, Vindasiute E, Puisys A, Linkeviciene L, Maslova N, Puriene A. The influence of the cementation margin position on the amount of undetected cement. A prospective clinical study. Clin Oral Implants Res. 2013;24(1):71-6.

47. Cha HS, Kim YS, Jeon JH, Lee JH. Cumulative survival rate and complication rates of single-tooth implant; focused on the coronal fracture of fixture in the internal connection implant. J Oral Rehabil. 2013;40(8):595-602.

48. Romeo E, Tomasi C, Finini I, Casentini P, Lops D. Implant-supported fixed cantilever prosthesis in partially edentulous jaws: a cohort prospective study. Clin Oral Implants Res. 2009;20(11):1278-85.

49. Levine RA, Clem DS 3rd, Wilson TG Jr, Higginbottom F, Solnit G. Multicenter retrospective analysis of the ITI implant system used for single-tooth replacements: results of loading for 2 or more years. Int J Oral Maxillofac Implants. 1999;14(4):516 20.

50. De Boever AL, Keersmaekers K, Vanmaele G, Kerschbaum T, Theuniers G, De Boever JA. Prosthetic complications in fixed endosseous implant-borne reconstructions after an observations period of at least 40 months. J Oral Rehabil. 2006;33(11):833-9.

51. Canullo L, Peñarrocha-Oltra D, Covani U, Botticelli D, Serino G, Penarrocha M. Clinical and microbiological findings in patients with peri-implantitis: a crosssectional study. Clin Oral Implants Res. 2016;27(3):376-82.

52. Dalago HR, Schuldt Filho G, Rodrigues MA, Renvert S, Bianchini MA. Risk indicators for Peri-implantitis. A cross-sectional study with 916 implants. Clin Oral Implants Res. 2017;28(2):144-50.

53. Serino G, Wada M. Non-surgical mechanical treatment of peri-implant mucositis: the effect of sub-mucosal mechanical instrumentation following supra-mucosal plaque removal. A 7-month prospective single cohort study. Eur J Oral Implantol. 2018;11(4):455-66.

54. Scholander S. A retrospective evaluation of 259 single-tooth replacements by the use of Brånemark implants. Int J Prosthodont. 2000;12(6):483-91.

55. Simon RL. Single implant-supported molar and premolar crowns: a ten-year retrospective clinical report. J Prosthet Dent. 2003;90(6):517-21.

56. Gibbard LL, Zarb G. A 5-year prospective study of implant-supported singletooth replacements. J Can Dent Assoc. 2002;68(2):110-6.

57. Sailer I, Zembic A, Jung RE, Siegenthaler D, Holderegger C, Hämmerle $\mathrm{CH}$. Randomized controlled clinical trial of customized zirconia and titanium implant abutments for canine and posterior single-tooth implant reconstructions: preliminary results at 1 year of function. Clin Oral Implants Res. 2009;20(3):219-25

58. Fugazzotto PA, Vlassis J, Butler B. ITI implant use in private practice: clinical results with 5,526 implants followed up to $72+$ months in function. Int $\mathrm{J}$ Oral Maxillofac Implants. 2004;19(3):408-12.

59. Maló P, de Araújo Nobre M, Borges J, Almeida R. Retrievable metal ceramic implant-supported fixed prostheses with milled titanium frameworks and all-ceramic crowns: retrospective clinical study with up to 10 years of follow-up. J Prosthodont. 2012;21(4):256-64

60. Andersson B, Taylor A, Lang BR, Scheller H, Schärer P, Sorensen JA, et al. Alumina ceramic implant abutments used for single-tooth replacement: a prospective 1- to 3-year multicenter study. Int J Prosthodont. 2001;14(5):432-8. 